\title{
Prediction of annual dengue incidence by hydro-climatic extremes for southern Taiwan
}

\author{
Hsiang-Yu Yuan ${ }^{1}$ - Tzai-Hung Wen ${ }^{2} \cdot$ Yi-Hung Kung ${ }^{3} \cdot$ Hsiao-Hui Tsou $^{3,4} \cdot$ Chun-Hong Chen ${ }^{3,5} \cdot$ Li-Wei Chen $^{4}$. \\ Pei-Sheng $\operatorname{Lin}^{3,4}$
}

Received: 12 June 2018 / Revised: 30 October 2018 / Accepted: 3 December 2018 / Published online: 24 January 2019

(C) ISB 2019

\begin{abstract}
Dengue is one of the most rapidly spreading mosquito-borne viral diseases in the world. An increase in the incidence of dengue is commonly thought to be a consequence of variability of weather conditions. Taiwan, which straddles the Tropic of Cancer, is an excellent place to study the relationship between weather conditions and dengue fever cases since the island forms an isolated geographic environment. Therefore, clarifying the association between extreme weather conditions and annual dengue incidence is one of important issues for epidemic early warning. In this paper, we develop a Poisson regression model with extreme weather parameters for prediction of annual dengue incidence. A leave-one-out method is used to evaluate the performance of predicting dengue incidence. Our results indicate that dengue transmission has a positive relationship with the minimum temperature predictors during the early summer while a negative relationship with all the maximum 24-h rainfall predictors during the early epidemic phase of dengue outbreaks. Our findings provide a better understanding of the relationships between extreme weather and annual trends in dengue cases in Taiwan and it could have important implications for dengue forecasts in surrounding areas with similar meteorological conditions.
\end{abstract}

Keywords Dengue outbreak $\cdot$ Dengue prediction $\cdot$ Extreme weather $\cdot$ Minimum temperature $\cdot$ Maximum rainfall

\section{Introduction}

Dengue is one of the world's most rapidly spreading mosquito-borne viral diseases, its incidence increasing 30fold during the last 50 years (WHO 2009). A recent study estimated that 390 million people are infected each year (Bhatt et al. 2013). The World Health Organization (WHO)

Pei-Sheng Lin

pslin@nhri.org.tw

1 Department of Biomedical Sciences, City University of Hong Kong, Kowloon Tong, Hong Kong

2 Department of Geography, National Taiwan University, Taipei City, Taiwan

3 National Mosquito-Borne Disease Control Research Center, National Health Research Institutes, 35 Keyan Road, Zhuna, Miaoli 350, Taiwan

4 Institute of Population Health Sciences, National Health Research Institutes, 35 Keyan Road, Zhuna, Miaoli 350, Taiwan

5 Institute of Infectious Diseases and Vaccinology, National Health Research Institutes, Zhuna, Taiwan recently warned that the number of dengue cases was increasing and about half of the world's population is now at risk (WHO 2017). The rapid increase of dengue incidence has been especially noteworthy in Southeast and East Asia. In years 2014 and 2015, record-high numbers of dengue cases occurred in Taiwan; at almost the same time, Japan experienced its first dengue fever (DF) outbreak in almost 70 years (Kutsuna et al. 2015). This phenomenon may indicate the rapid spread of dengue virus (DENV) into counties with temperate climates. An increase in the incidence of dengue is commonly thought to be a consequence of variability of weather conditions (Hales et al. 2002; Morin et al. 2013). Several models have demonstrated the feasibility of using weather data to forecast dengue outbreaks (Morales et al. 2016; Xu et al. 2017). Among weather-related factors, temperature and rainfall have been found to be two most significant weather parameters, with both having major influences on DENV ecology through Aedes albopictus and Aedes aegypti. For example, temperature has been demonstrated to affect dengue incidence directly through the development of the dengue mosquito (Yang et al. 2009a), and rainfall also has an impact on the availability of mosquito habitats (Hoeck et al. 
2003). While changes of temperature do affect the mosquito population dynamics, the changes can also affect extrinsic incubation period (EIP) of DENV. An infected mosquito is infectious in a shorter EIP at higher temperatures (Mutheneni et al. 2017). While precipitation obviously can provide standing water and mosquito breeding sites, studies (see, e.g., Beebe et al. 2009) have also shown that drought periods can increase the likelihood of water storage around the home in open containers or the formation of standing pools in nearly dry streambeds, which also provide breeding sites. Heavy rainfall can flush out standing water, possibly reducing mosquito populations for a short time. The relationship between dengue and precipitation can thus be complicated. Since variability of climate could greatly influence dengue incidence, further understanding of the association between extreme weather conditions and dengue incidence would allow early prediction and prevention of DENV transmission.

Taiwan is an isolated island located in the East Asian monsoon region, with part of Taiwan belonging to the northern temperate zone. Two extreme environmental situations for the growth of mosquitoes in Taiwan are caused by weather conditions: relatively high temperatures and humidity in summer are ideal for the dissemination and growth of the Aedes mosquitoes, while low temperatures in winter (due to continental cold air from China) cause insect diapause. The amount of rainfall in Taiwan varies greatly by month in each year. Drought, monsoon, and typhoon events greatly affect the frequency and amount of extreme rainfall. The variability of climate could largely affect DENV transmission in Taiwan. Particularly, unprecedented consecutive large dengue outbreaks in Taiwan in the years 2014 and 2015 gained great attention among public health experts for the risk of DENV. Most of the dengue incidence occurred in Kaohsiung, southern Taiwan's largest city. During years 2005-2013, a total of 5334 cases occurred in this city. However, in years 2014 and 2015, there were 15,085 and 19,975 reported DF cases, respectively, identified in Kaohsiung.

Since Taiwan is an island with most DF epidemic initiated from imported cases, a greater understanding of the relationship between weather conditions and DF cases in Taiwan could help other countries prevent their own DF outbreaks. Recent literature has found evidence that climate change could drive dengue transmission ( $\mathrm{Xu}$ et al. 2017). Extreme weather such as very heavy rainfall events could represent large variability of conditions. Although several early-warning systems for dengue outbreaks have been developed to facilitate the public health response (Shi et al. 2016), most associated studies used linear regression models with a time lag to forecast weekly dengue incidence of an endemic (Hii et al. 2012). To visualize the relationship between DF cases and climatic data in Taiwan, we use Figs. 1 and 2 to show the monthly mean number of cases and observed numbers of yearly cases,
Fig. 1 Average numbers of monthly dengue cases in Kaohsiung City from years 2005 to 2016

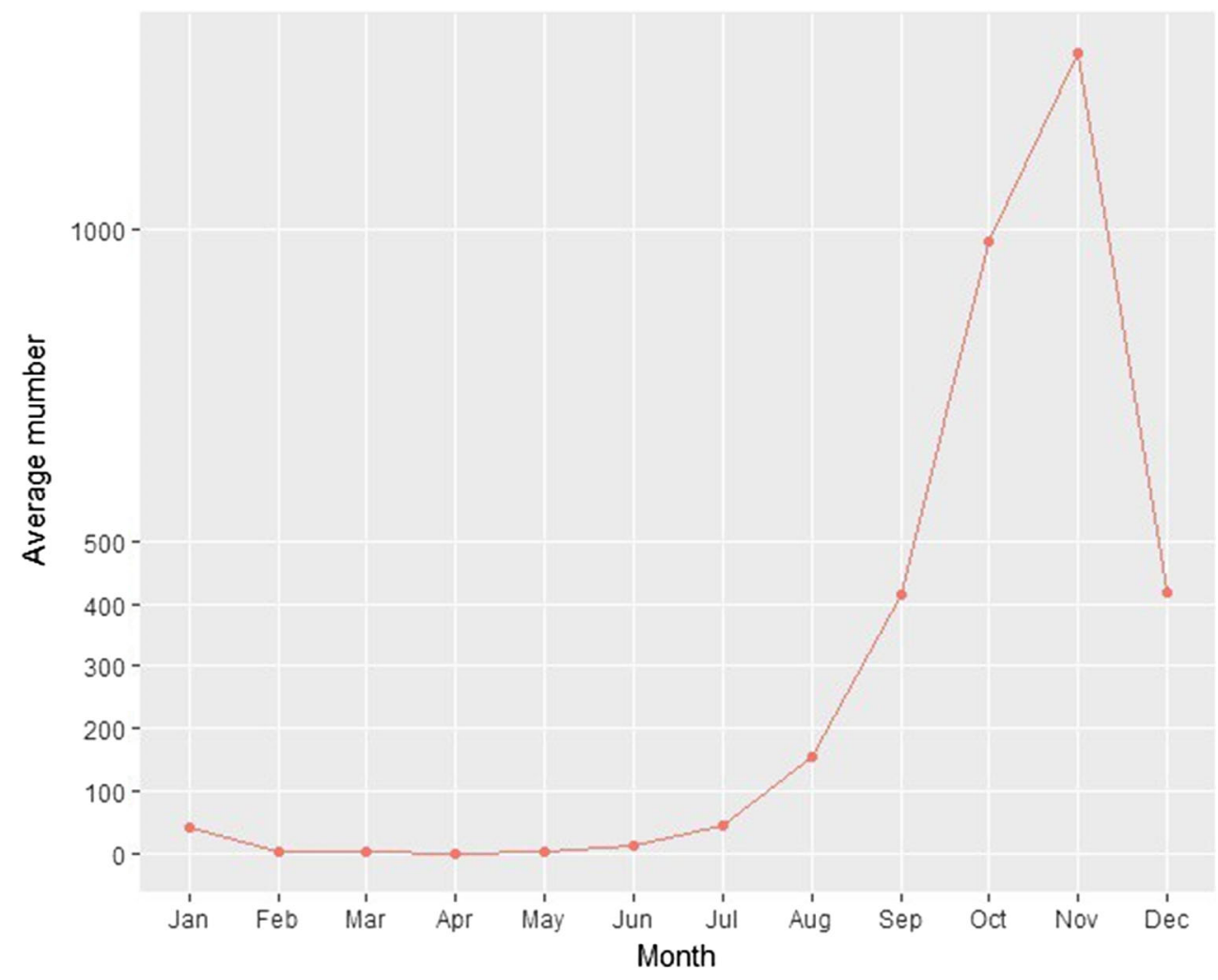


respectively. As can be seen from Fig. 1 for average numbers of monthly dengue cases in Taiwan, dengue epidemics commonly do not start until after August, contrary to the situation in other tropical countries, where dengue epidemics can occur almost any time of the year. Also, Fig. 2 shows that in Taiwan, between-year dengue incidences varied greatly. So, a prediction model that can use climatic information prior to August to forecast whether there will be a DF outbreak in the given year is primarily important. With this model, public health policy makers can obtain an early prediction, before the epidemic peaks, for annual DF cases in order to better control and prevent dengue.

At this point, Khalid and Ghaffar (2015a, b) developed useful models for association of environmental risk factors and dengue outbreaks in a study of dengue transmission in Pakistan. Since the effect of time lag on dengue incidence is complicated and varied, we use monthly climatic data from only January to August to forecast the annual trend of dengue cases. Contrary to the real-time monitoring and prediction system that relies on estimating weekly dengue cases, our approach allows us to capture the impacts of weather from different months without a constraint on the unique optimal lag period. Therefore, the purposes of the study are to clarify the role of monthly extreme weather conditions in annual dengue incidence and to develop an epidemic prediction model for forecasting annual trend of dengue cases.
This paper is then organized as follows. In the "Materials and methods" section, we develop a Poisson regression model for prediction based on data from the years 2005 to 2016 in Kaohsiung, Taiwan. Since extreme values of temperature and rainfall could be better to capture variability of climatic data, two extreme weather covariates, minimum temperature and maximum cumulative rainfall, are used for the prediction models. A variable selection procedure by Akaike Information Criterion (AIC) is then adapted for model selection. We use a leave-one-out cross validation method to evaluate performance of the proposed model. In the "Analysis result" section, we present the results of our analysis, followed by a discussion in the "Discussion" section.

\section{Material and methods}

\section{Data collection}

Annual dengue cases occurred in Kaohsiung from years 2005 to 2016 were obtained from the Taiwan Centers of Disease Control (https://www.cdc.gov.tw/rwd/en). The annual number of dengue cases was defined as the total reported cases whose disease onset date was between April 1 in a given year and March 31 the following year. Imported cases were excluded to ignore the factors of infection that related to traveling. Hourly temperature and
Fig. 2 Observed numbers of yearly dengue cases in Kaohsiung City from years 2005 to 2016

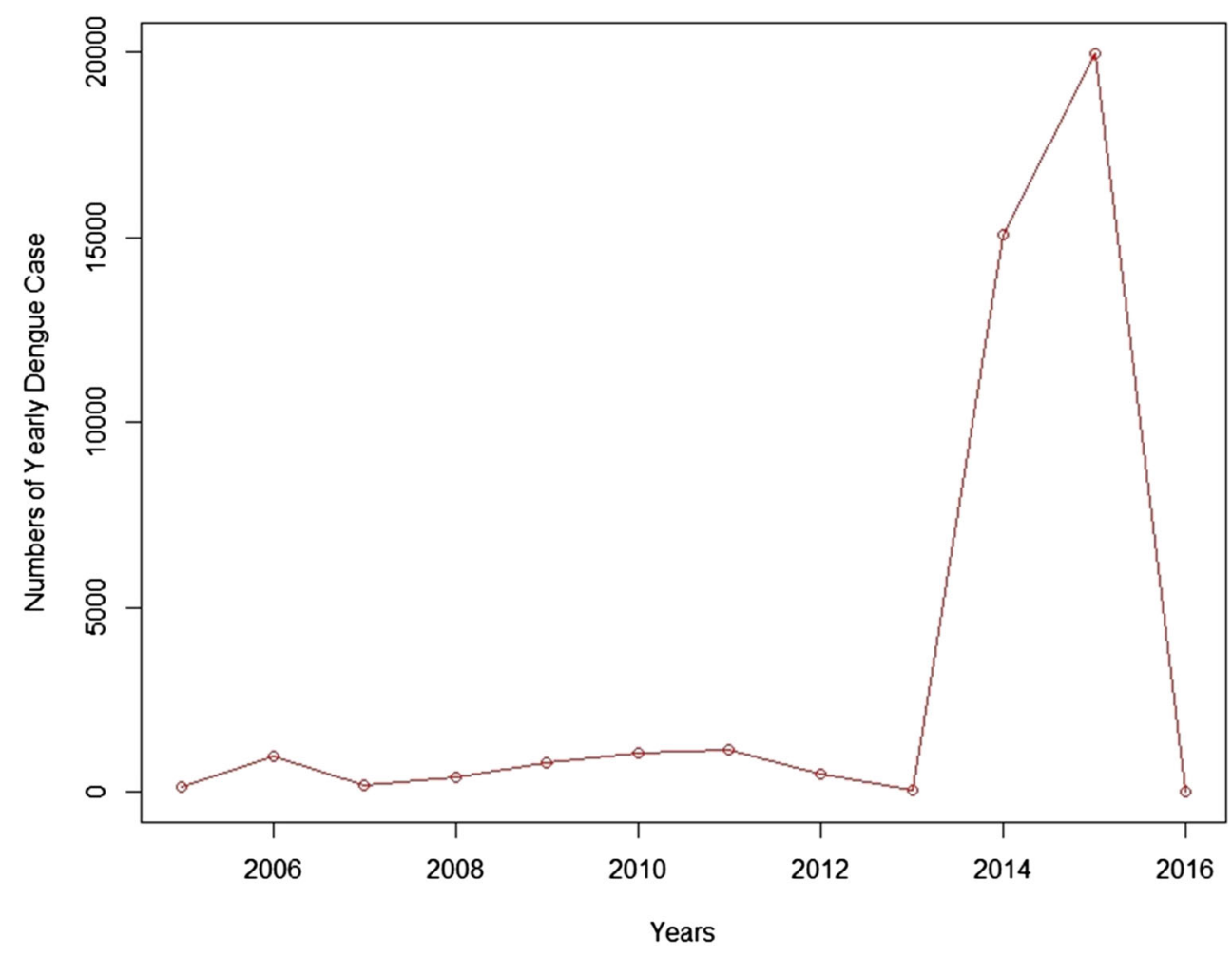


rainfall were collected from the Central Weather Bureau of Taiwan. In this study, we defined daily temperature as the average of hourly temperature per day, monthly average temperature as the mean of daily temperature in a given month, monthly minimum temperature as the lowest daily temperature in a given month, daily 24 -h accumulated rainfall as the summation of the hourly rainfall, the monthly average 24 -h rainfall as the mean of daily 24 -h accumulated rainfall, and the monthly maximum $24-\mathrm{h}$ rainfall as the maximum daily 24 -h accumulated rainfall in a given month.

\section{Choice of explanatory variables}

DF cases are frequently reported between late summer and early winter in Taiwan. We picked monthly minimum temperature and maximum 24-h rainfall because these two climatic factors have been recently demonstrated to be the significant measures to dengue fever variations (Chien and Yu 2014). Figure 3 shows the temperature and rainfall data by box plots, with the monthly (average and minimum) temperatures in Fig. 3a and monthly (average and maximum) rainfalls in Fig. 3b. A quick comparison of Figs. 1 and 3 reveals that the pattern of dengue transmission coincides largely with the monthly temperature and rainfall, with few months' delay.

Furthermore, as can be seen from Fig. 3a, the monthly minimum temperature exhibited a larger variation (as represented by quartiles in box plots) than the average temperature for both within and between months. Comparing with the monthly average 24-h rainfall in Fig. 3b, the maximum 24-h rainfall demonstrated a greater amount of value and a larger variability. Therefore, to better catch the impact of weather conditions for dengue fever transmission, we use minimum temperature and maximum rainfall as indicators of extreme weather in our study.

\section{Poisson regression model with AIC}

We now develop a Poisson regression model to quantify the relationship between the number of dengue cases and the monthly maximum rainfall and minimum temperature, using data from years 2005 to 2016 . Let $Z_{i, j}, R_{i, j}$, and $T_{i, j}$ denote the corresponding number of dengue cases, the largest daily precipitation, and the lowest temperature in $i$ th month of the $j$ th year, respectively, $i, j=1, \ldots, 12$. (We set year 2005 to be the first year, and so on.) Let

$Y_{j}=\sum_{i=4}^{12} Z_{i, j}+\sum_{i=1}^{3} Z_{i, j+1}$

denote the total number of dengue cases from April of the $j$ th year to the end of March of the year $(j+1)$. In this paper, $Y_{j}$ is defined as the number of annual cases. Let $\mu_{j}=E\left(Y_{j}\right)$ denote an expected number of annual cases.

Since $Y_{j}$ follows a Poisson distribution, we fit the expected incidence $\mu_{j}$ by a log-linear model. As can be seen in Fig. 1, the total number of cases from April to August occupied only a very small portion of the whole year. Also, Eq. (1) tells us that the total number of cases from January to March can be ascribed to a continuation of the previous year's outbreak. So, predicting the number of annual cases is approximately equal to predicting the number of cases after August. Let $\mu_{j}^{*}$ denote an expected number of total cases from September of the $j$ th year to March of the year $(j+1)$. Then, $\mu_{j}^{*} \approx \mu_{j}$.

The Poisson prediction model based on climatic data can thus be given by

$\log \left(\mu_{j}\right) \approx \log \left(\mu_{j}^{*}\right)=\alpha+\sum_{i \in I} R_{i, j} \beta_{i}+\sum_{i \in J} T_{i, j} \gamma_{i}$,

where $\beta_{i}$ and $\gamma_{i}$ are the corresponding regression coefficients associated with the $i$ th month rainfall data $R_{i}=\left(R_{i}\right.$, $\left.{ }_{1}, \ldots, R_{i, 12}\right)^{\prime}$ and temperature data $T_{i}=\left(T_{i, 1}, \ldots, T_{i, 12}\right)^{\prime}$, respectively. Also, notations $I$ and $J$ denote index sets for months that are selected into the model by a model selection procedure. (The final model is shown in (4).) Since the sample size is small, a corrected version of AIC (AICc) (Hurvich and Tsai 1991) is used as a model selection criterion. We use a forward selection procedure by sequentially adding climatic variables, starting from the most significant one, into the model.

\section{Leave-one-out cross validation}

Leave-one-out cross validation is used to measure the fit of the model (Stone 1974). In each iteration, one sample (a year) is left out (for a validation set), and the rest of data (a training set) is used to fit the model. The fitted model by the training set is then used to predict the number of DF cases for the validation set. The procedure is repeated until every year has been used for a validation set. For linear models, under mild assumptions, the expected ratio of mean squared error (MSE) between the validation and training sets should be less than $(n+p+1) /$ $(n-p-1)$ (Trippa et al. 2015), where $n$ and $p$ denote the sample size and number of parameters, respectively. That is, the criterion used to examine the goodness of fit for the prediction model is

$F=\frac{\mathrm{MSE}_{\mathrm{Va}}}{\mathrm{MSE}_{\mathrm{Tr}}}<\frac{n+p+1}{n-p-1}$,

where $\mathrm{MSE}_{\mathrm{Va}}$ and $\mathrm{MSE}_{\mathrm{Tr}}$ denote the mean squared errors for the validation and training sets, respectively. To see why the criterion (3) is reasonable for the leave-one-out procedure, we note that in (3), the term of $n-p-1$ can be regarded as the 
Fig. 3 Box plots for monthly climatic data in Kaohsiung, 2005-2016. a Temperatures by month: Red box indicates minimum temperature and blue box denotes average temperature. b Daily rainfall: Red box denotes maximum 24-h rainfall and blue box represents average $24-\mathrm{h}$ rainfall a

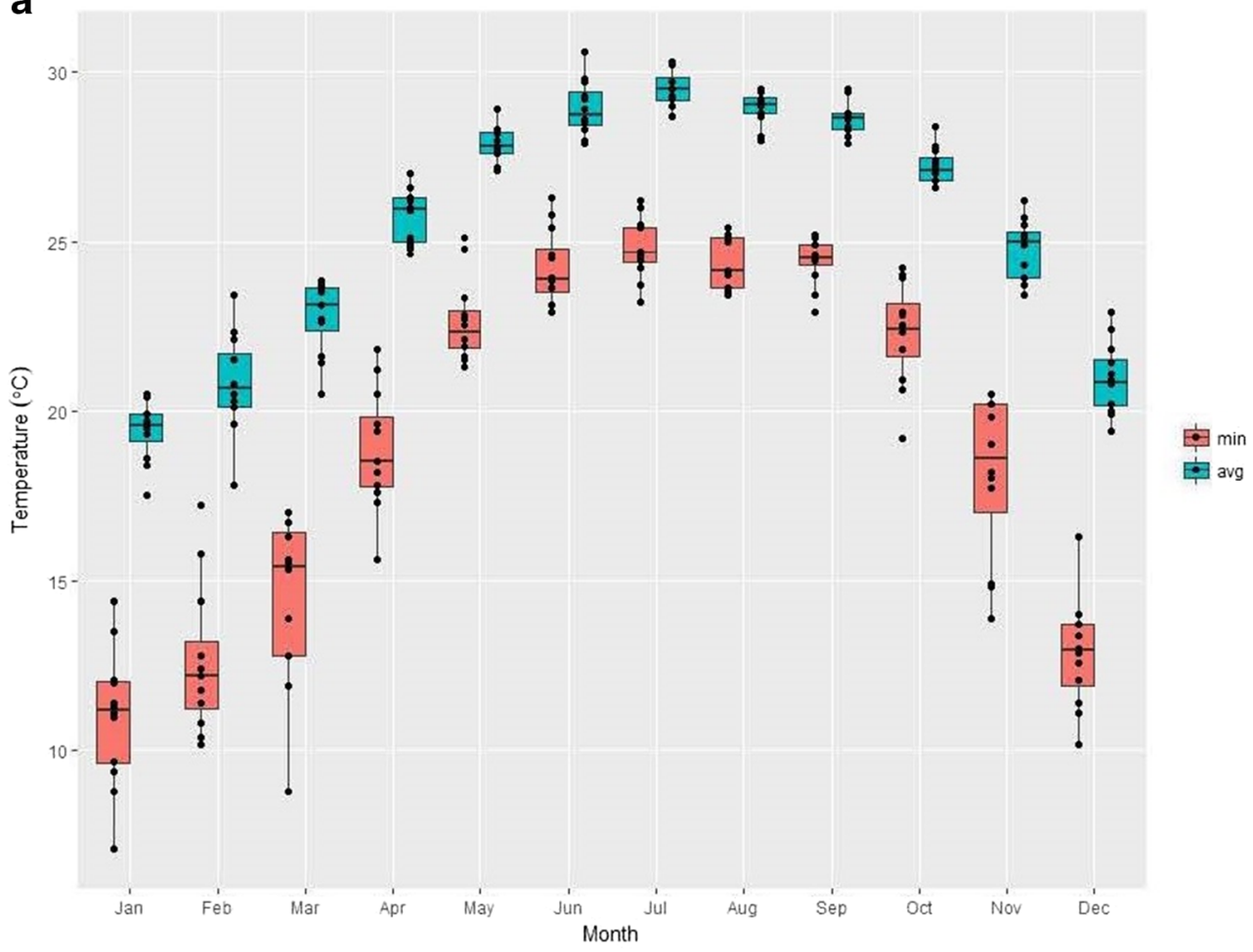

b

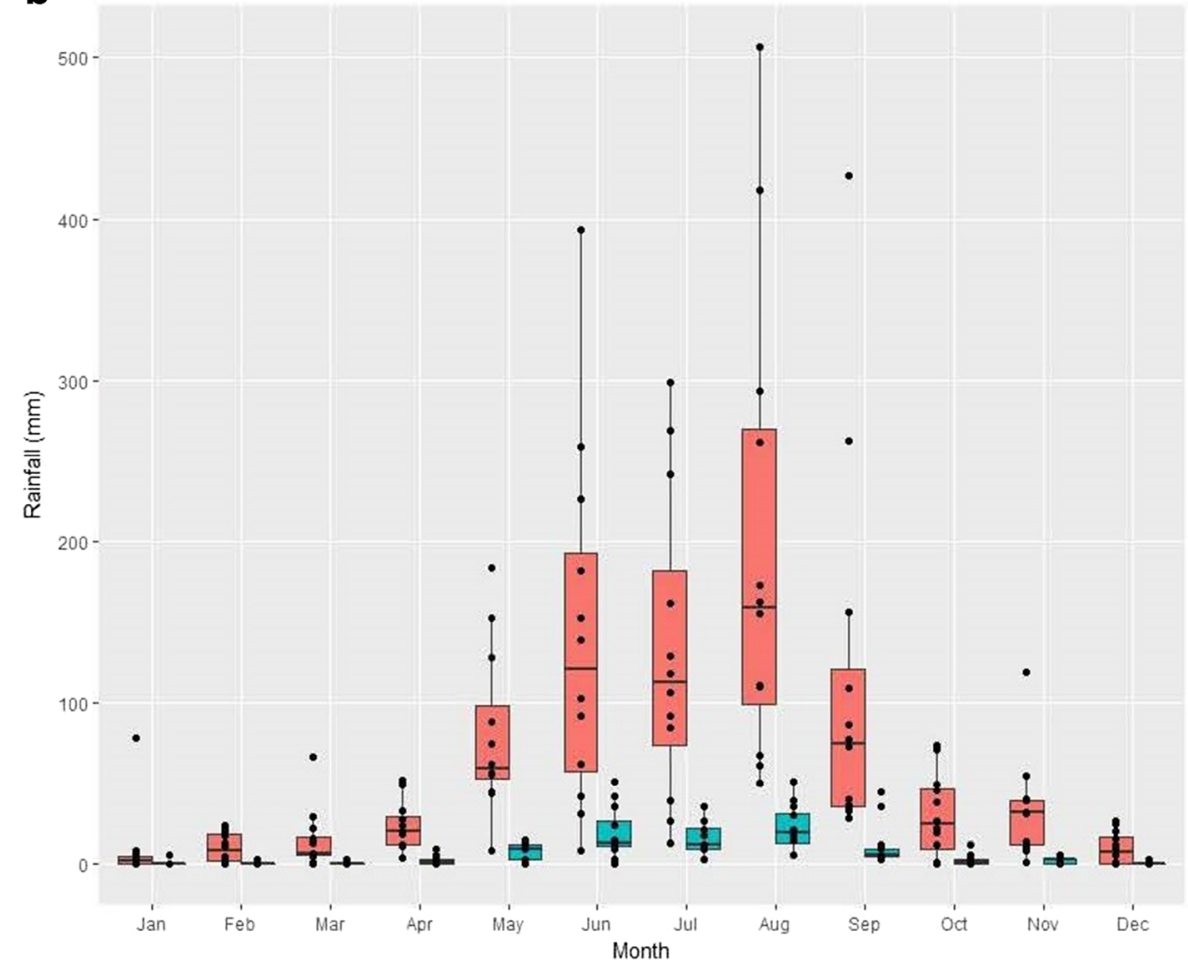

审 max 审 avg degrees of freedom for the linear model in the training set. (The sample size used in the training set is $n$-1.) On the other hand, from the Bayesian aspect, the term of $n+p+1$ represents the degrees of freedom for the validation set. (For the model in the validation set, all parameters are estimated from the training set.) Therefore, when the model is correctly specified, we would expect that $\mathrm{MSE}_{\mathrm{Va}}<(n+p+1) \mathrm{MSE}_{\mathrm{Tr}} /(n-$ $p-1$ ), which leads to (3). 


\section{Analysis result}

\section{Prediction model for annual dengue cases}

We used the forward selection to choose suitable predictors from 16 climatic variables, $T_{1}, \ldots, T_{8}, R_{1}, \ldots, R_{8}$ (monthly minimum temperatures and maximum rainfalls from January to August), for the Poisson model (2). Table 1 shows the model selection result by the forward AICc procedure. The final fitted Poisson model for prediction, with standard errors for estimated coefficients in parentheses, is given by

$$
\begin{aligned}
\log \left(\mu_{j}\right)= & -51.9(1.17)-0.44(0.04) T_{2, j}+1.39(0.08) T_{5, j} \\
& +0.87(0.03) T_{6, j}+0.75(0.05) T_{7, j} \\
& -0.13(0.01) R_{1, j}-0.27(0.01) R_{2, j}-0.05(0.002) R_{4, j} \\
& -0.03(0.0005) R_{5, j}-0.003(0.0004) R_{8, j} .
\end{aligned}
$$

Model (4) contains four minimum temperature and five maximum rainfall variables. Note that as discussed in model (1), $\log \left(\mu_{j}^{*}\right) \approx \log \left(\mu_{j}\right)$. Some remarks for the forward selection procedure are given below. First, as can be seen from Table 1, there was only one climatic variable in the first model, and in the final step, nine climatic variables were selected into the model. So, in the forward selection procedure, all Poisson model satisfied the condition that the sample size $(n=12)$ was greater than the number of estimated coefficients $p$ (at most ten parameters including the intercept). Second, for the climatic data, we note that multi-collinearity may exist due to association between monthly temperatures and rainfalls. However, the forward selection procedure could mitigate the multicollinearity issue since one predictor can be added into a model only when the predictor can significantly reduce the deviance. That is, if two climatic variables had strong association, then only one of them can possibly be selected.
Table 2 shows the estimated result by model (4) with twosided confidence intervals. As can be seen from Table 2, the observed numbers of each year (except year 2016 with very few cases), including two large consecutive outbreaks during years 2014 and 2015, are all in the 95\% confidence interval of the estimated numbers. The rapid increase from a very low number of cases in year 2013 (65 cases) to extremely high during year 2014 ( 15,085 cases) and year 2015 (19,975 cases) is recovered as the estimated number cases 81 (65-98), 15,076 $(14,843-15,340)$ and $19,982(19,673-20,230)$.

Model (4) demonstrates that the number of the dengue transmission has a positive relationship with the minimum temperature predictors in February and months during the early summer, such as May, June, and July, while, interestingly, the number of the dengue transmission has a negative relationship with all the maximum 24-h rainfall predictors in January, February, April, May, and August. Most of these months are distributed across before and during the early epidemic phase of dengue outbreaks. These positive impacts of minimum temperature during the early summer and negative impacts of maximum rainfall before and during the early epidemic phase on dengue transmission are consistently shown in other models during variable selection using AICc (see Table 1).

\section{Cross validation for the prediction model}

To evaluate the prediction performance of model (4), the leave-one-out cross validation method is performed. In each validation iteration, data set of a year was removed and we reestimated the expected number of annual cases in this year. Let $\hat{\mu}_{j}^{(-j)}$ denote the predicted number of annual cases for the $j$ th year with the $j$ th year as a validation set. Table 3 shows the leave-one-out validation result with $\hat{\mu}_{j}^{(-j)}$ shown in the diagonal number (in italics). As can be seen from Table 3, the prediction model is still able to predict large outbreaks in years
Table 1 Forward selection results in various stages by $\mathrm{AICc}$ for the Poisson model. $T_{i}$ and $R_{i}$ represent the minimum temperature and maximum rainfall, respectively, for the $i$ th month

\begin{tabular}{lllllllllll}
\hline Intercept & \multicolumn{1}{l}{ Variables } \\
\cline { 2 - 9 } & $T_{6}$ & $R_{5}$ & $R_{1}$ & $R_{8}$ & $R_{2}$ & $R_{4}$ & $T_{7}$ & $T_{5}$ & $T_{2}$ & AICc \\
\hline-22.0 & 1.21 & & & & & & & & & 38,940 \\
-22.7 & 1.31 & -0.03 & & & & & & & & 12,492 \\
-21.5 & 1.27 & -0.03 & -0.06 & & & & & & & 3913 \\
-18.9 & 1.24 & -0.04 & -0.12 & -0.005 & & & & & & 1861 \\
-26.4 & 1.58 & -0.04 & -0.08 & -0.006 & -0.06 & & & & & 1071 \\
-24.8 & 1.53 & -0.04 & -0.07 & -0.006 & -0.08 & -0.03 & & & & 919 \\
-26.0 & 1.38 & -0.03 & -0.05 & -0.006 & -0.08 & -0.03 & 0.19 & & & 807 \\
-49.9 & 0.91 & -0.03 & -0.06 & -0.007 & -0.19 & -0.06 & 1.04 & 0.69 & & 383 \\
-51.9 & 0.87 & -0.03 & -0.13 & -0.003 & -0.27 & -0.05 & 0.75 & 1.39 & -0.44 & 366 \\
\hline
\end{tabular}


Table 2 Comparison of the observed and estimated annual numbers of dengue cases in Kaohsiung. Estimated values are from Poisson regression with maximum precipitation and minimum temperature. CI (lower) and CI (upper) denote lower and upper bounds, respectively, for confidence intervals

\begin{tabular}{|c|c|c|c|c|c|c|c|c|c|c|c|c|}
\hline & \multicolumn{12}{|c|}{ Year } \\
\hline & 05 & 06 & 07 & 08 & 09 & 10 & 11 & 12 & 13 & 14 & 15 & 16 \\
\hline Observed & 126 & 958 & 168 & 422 & 787 & 1047 & 1165 & 504 & 65 & 15,085 & 19,975 & 3 \\
\hline Estimate & 105 & 924 & 161 & 487 & 804 & 1082 & 1116 & 487 & 81 & 15,076 & 19,982 & 0 \\
\hline CI (lower) & 89 & 875 & 137 & 454 & 752 & 1021 & 1063 & 446 & 65 & 14,843 & 19,673 & 0 \\
\hline CI (upper) & 126 & 984 & 186 & 523 & 862 & 1151 & 1159 & 528 & 98 & 15,340 & 20,230 & 1 \\
\hline
\end{tabular}

2014 and 2015 and extremely low cases in years 2005, 2013, and 2016. Let $\hat{\mu}_{i}^{(-j)}$ denote the estimated number of annual cases for the $i$ th year with data of the $j$ th year being removed. We computed the mean squared errors for the training and validation sets by $\mathrm{MSE}_{\mathrm{Va}}=\sum_{j=1}^{12} \sum_{i \neq j}\left(\log \left(\hat{\mu}_{i}^{(-j)}\right)-\log \left(\mu_{i}\right)\right)^{2}$ $/ 132$ and $\mathrm{MSE}_{\mathrm{Tr}}=\sum_{j=1}^{12}\left(\log \left(\hat{\mu}_{j}^{(-j)}\right)-\log \left(\mu_{j}\right)\right)^{2} / 12$. By (3), we then have $\mathrm{MSE}_{\mathrm{Va}}=2.21$ and $\mathrm{MSE}_{\mathrm{Tr}}=2.11$ with an $F$-ratio at a value of 1.16 , which is far below the threshold value of $11(=(n+p+1) /(n-p-1))$. The ratio of mean squared errors indicates that prediction model works very well.

\section{Relationship between climate predictors and large outbreaks}

To investigate whether a relationship between the climate predictors and large outbreaks exists, we used Fig. 4 to further explore monthly weather conditions by a comparing the climatic data of years 2014-2015 with those of other years. As can be seen in Fig. 4a, years 2014 and 2015 had minimum temperatures significantly higher than other years in June and July, in which months the predicted model (4) demonstrates positive effects on $T_{6}$ and $T_{7}$. In Fig. $4 \mathrm{~b}$, the maximum rainfalls in the outbreak years (2014 and 2015) are also found to be lower than those in the other years in most of the months, while the maximum rainfall in these months has been demonstrated to have a negative relationship to dengue cases. These results may provide further evidence for the validation of model (4).

\section{Discussion}

Taiwan is located across the Tropic of Cancer and is geographically surrounded by China, Japan, and some Southeast Asian countries. While dengue endemic occurred in most Southeast Asian countries (Shepard et al. 2013), few DF outbreaks have been seen in East Asian countries. However, in the recent years, DF has diffused to regions and countries in East Asia that had rarely or never experienced dengue outbreaks before, for example, China's Shandong and Zhejiang provinces, and Japan (Bhatt et al. 2013; Kutsuna et al. 2015; Lai et al. 2015).

Table 3 Leave-one-out cross validation results for dengue cases from 2005 to 2016, with maximum precipitation and minimum temperature. Numbers in italics indicate the predicted value by the leave-one-out method

\begin{tabular}{lcccccccccccc}
\hline & 2005 & 2006 & 2007 & 2008 & 2009 & 2010 & 2011 & 2012 & 2013 & 2014 & 2015 & 2016 \\
\hline 2005 & 48 & 967 & 168 & 447 & 792 & 1057 & 1109 & 490 & 70 & 15,115 & 19,960 & 4 \\
2006 & 119 & 682 & 166 & 473 & 798 & 1070 & 1083 & 482 & 75 & 15,117 & 19,960 & 3 \\
2007 & 126 & 967 & 8 & 448 & 792 & 1058 & 1107 & 490 & 70 & 15,115 & 19,960 & 4 \\
2008 & 117 & 934 & 165 & 602 & 789 & 1051 & 1204 & 511 & 67 & 15,050 & 19,994 & 1 \\
2009 & 123 & 947 & 167 & 418 & 4077 & 1047 & 1189 & 509 & 65 & 15,067 & 19,984 & 2 \\
2010 & 122 & 946 & 167 & 418 & 787 & 2262 & 1192 & 510 & 65 & 15,064 & 19,986 \\
2011 & 108 & 923 & 162 & 458 & 798 & 1069 & 1041 & 499 & 75 & 15,056 & 19,992 \\
2012 & 110 & 924 & 163 & 446 & 795 & 1063 & 1182 & 283 & 72 & 15,051 & 19,994 & 0 \\
2013 & 122 & 946 & 167 & 418 & 787 & 1047 & 1191 & 509 & 364 & 15,065 & 19,985 \\
2014 & 106 & 928 & 162 & 492 & 804 & 1084 & 1102 & 483 & 81 & 14,167 & 19,977 & 1 \\
2015 & 107 & 931 & 162 & 493 & 804 & 1084 & 1096 & 482 & 81 & 15,090 & 23,685 & 1 \\
2016 & 117 & 953 & 165 & 479 & 800 & 1074 & 1081 & 481 & 77 & 15,114 & 19,961 & 0 \\
Observed & 126 & 958 & 168 & 422 & 787 & 1047 & 1165 & 504 & 65 & 15,085 & 19,975 & 3 \\
\hline
\end{tabular}


Fig. 4 Comparisons of climatic data between outbreak years (years 2014 and 2015) and other years. Red and blue lines denote average values of monthly climatic variables for outbreak years and other years, respectively. a Minimum temperature. b Maximum rainfall a

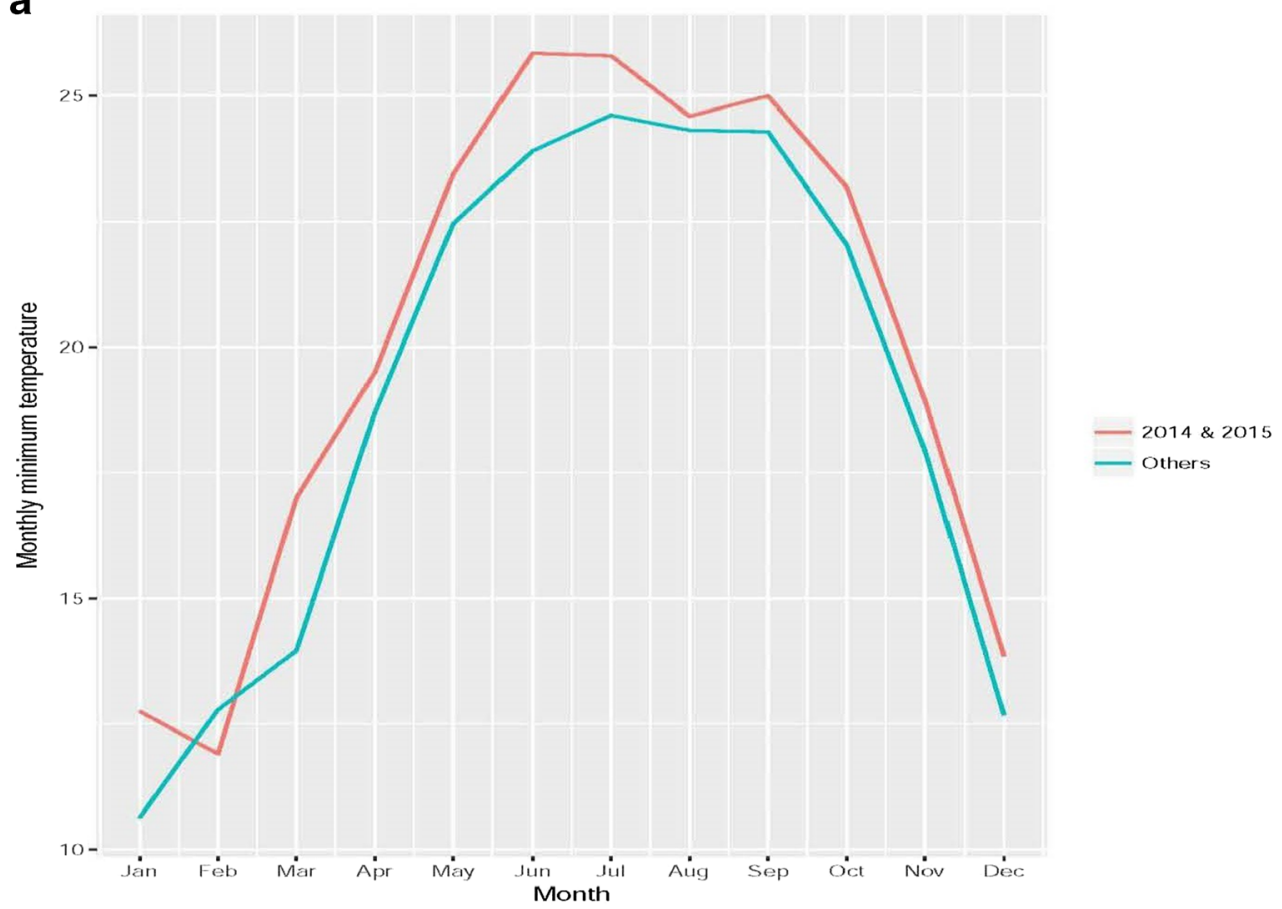

b

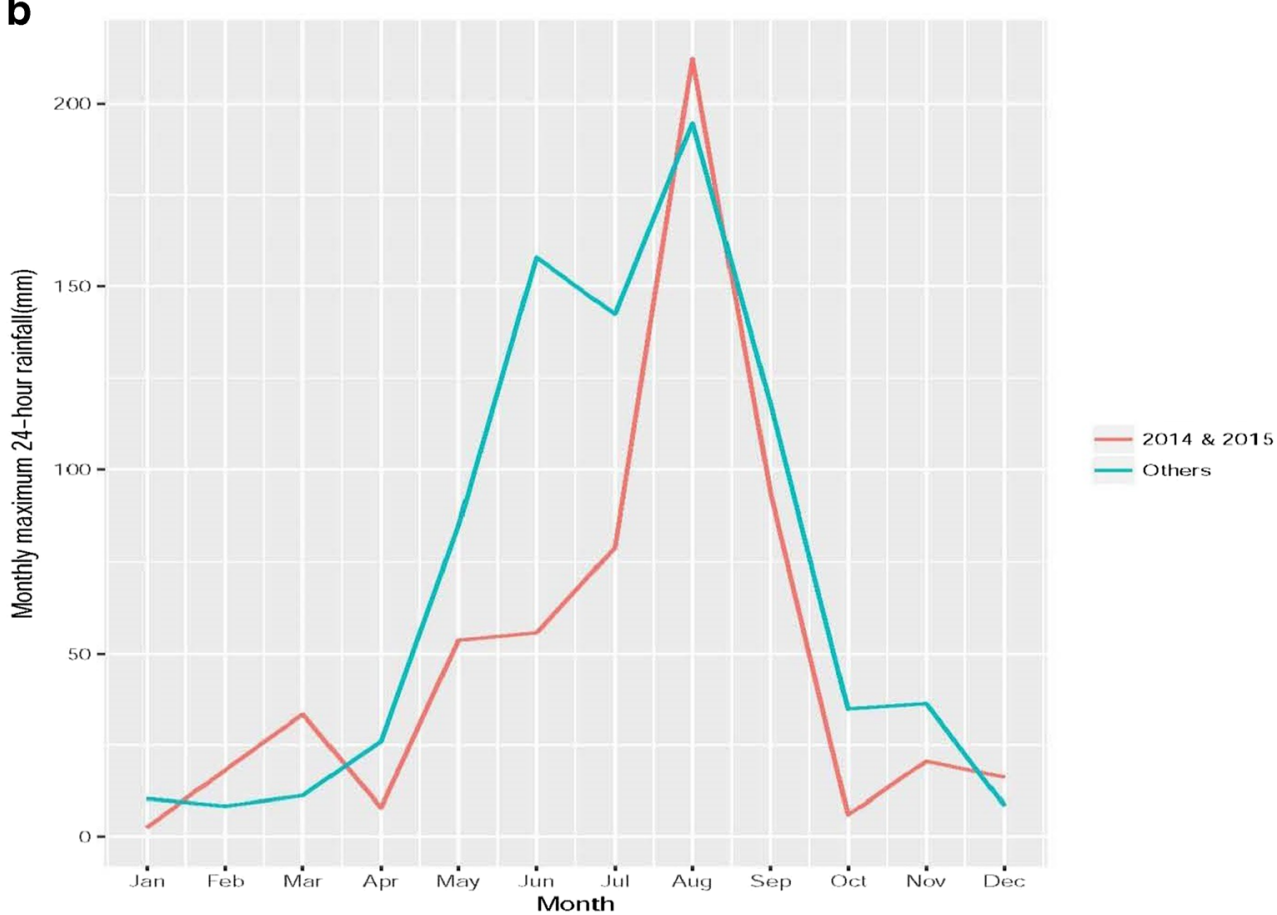

An understanding of the relationships between extreme weather and annual trends in dengue cases in Taiwan could have important implications for dengue forecasts in surrounding areas.

In this paper, we built a Poisson regression model by using the corrected AIC to forecast annual dengue cases. We find that the two extreme weather covariates, monthly minimum temperature and maximum 24-h rainfalls, are able to explain the large consecutive outbreaks as well as other seasonal epidemics in Taiwan, which provides significant indices for early warning of dengue epidemics. Previous studies have shown that a temperature higher than $27^{\circ} \mathrm{C}$ is optimal for the vital activities (e.g., biting) of the mosquito Aedes aegypti (Aragão 1939). Therefore, the monthly minimum temperature can significantly capture minimum requirement for dengue vector activities. However, the death rate of female mosquito 
significantly increases if temperature is higher than $33{ }^{\circ} \mathrm{C}$ (Yang et al. 2009b). The monthly maximum temperature is often higher than $33^{\circ} \mathrm{C}$ from April to November annually in southern Taiwan. It could be the reason the maximum temperature cannot significantly capture the annual trend of dengue incidence. Specifically, in the proposed model, the minimum temperatures in early summer from May to July are positively associated with the large outbreaks in Kaohsiung in 2014 and 2015. The impact of minimum temperature on dengue transmission proposed from our model is consistent with a previous report that a higher average temperature is linked to the increase of dengue with 2-3-month delayed effects, using dengue cases during the large outbreaks in years 2014 and 2015 (Wang et al. 2016).

However, one interesting point in the prediction model is that maximum 24-h rainfall was negatively associated with annual dengue cases. Although many studies have demonstrated the positive effects of rainfall on dengue transmission (Wang et al. 2016), some other works (Yu et al. 2016) have suggested that when the amount of rainfall was extremely high, the increase in rainfall could reduce the relative risk. One possible explanation for this phenomenon is that an excess amount of rainfall could destroy mosquito habitats and wash away mosquito eggs and larvae (Seidahmed and Eltahir 2016). There were studies showing that the flushing flow has a negative impact on the dengue transmission in urban areas, where water flows downward in the urban areas and drains out towards the streams or sea. Because most of the mosquito species tolerate the slow flowing or stagnant water, the heavy rains with flushing flows can decrease the dengue incidence (Khalid and Ghaffar 2015a). Therefore, the mosquito life cycle could be maintained only when the rainfall is in the proper amount. Since the mosquito lifespan ranges from 2 weeks to a month, maximum rainfall per month could be an important parameter to determine whether the mosquito life cycle is maintained. Another interesting point for the prediction model is that the minimum temperature in February had a negative effect for annual dengue cases. To explore a possible reason for this phenomenon, we observe Fig. 4a to notice that minimum February temperatures in years 2014 and 2015 were below than those in the other years. This might provide one reason to explain why the predominant strains of DENV in years 2014 (DENV-1) and 2015 (DENV-2) were different, by the serotyping study in Wang et al. (2016). Nevertheless, a further study for the negative effect of minimum February temperature on dengue cases should be needed.

Some limitations of the study are discussed here. First, the issue of small sample size exists in our Poisson regression model, although the small-sample-size corrected version of AIC was used for variable selection. One possible extension for the proposed model is to use monthly dengue case data. However, as mentioned in the previous discussion, monthly delayed effects may still exist for prediction of monthly DF cases. Second, the consequences of human intervention, including disease control, between different years are not measured to count possible impacts on annual incidences. Our results indicate rainfall could reduce numbers of the vectors. How amount of precipitation affects mosquito population dynamics remains to be studied.

Future work includes studies for identifying a possible cycle of DF epidemics in Kaohsiung. Due to cross-protective immunity among different DENV serotypes, an irregular pattern of epidemics with 8-10-year oscillation circles has occurred (Adams et al. 2006; Wearing and Rohani 2006). Therefore, both climate changes and DENV evolution under herd immunity would contribute to the epidemic patterns. Thus, to develop a model that incorporates seroconversion with cross-protection of individuals (Nagao and Koelle 2008) and the mechanism of how weather affects mosquito behaviors and life cycles (Xu et al. 2017) would be needed and may further increase the accuracy of dengue forecasts.

\section{References}

Adams B, Holmes EC, Zhang C, Mammen MP, Nimmannitya S, Kalayanarooj S, Boots M (2006) Cross-protective immunity can account for the alternating epidemic pattern of dengue virus serotypes circulating in Bangkok. Proc Natl Acad Sci 103(38):14234 14239

Aragão HB (1939) Mosquitoes and yellow fever virus. Mem Inst Oswaldo Cruz 34(4):565-581

Beebe MW, Cooper RD, Mottram P, Sweeney AW (2009) Australia's dengue risk driven by human adaptation to climate change. PLoS Negl Trop Dis 3(5):e429

Bhatt S, Gething PW, Brady OJ, Messina JP, Farlow AW, Moyes CL, Drake JM, Brownstein JS, Hoen AG, Sankoh O, Myers MF, George DB, Jaenisch T, Wint GR, Simmons CP, Scott TW, Farrar JJ, Hay SI (2013) The global distribution and burden of dengue. Nature 496(7446):504-507

Chien LC, Yu HL (2014) Impact of meteorological factors on the spatiotemporal patterns of dengue fever incidence. Environ Int 73:46-56

Hales S, De Wet N, Maindonald J, Woodward A (2002) Potential effect of population and climate changes on global distribution of dengue fever: an empirical model. Lancet 360(9336):830-834

Hii YL, Zhu H, Ng N, Ng LC, Rocklöv J (2012) Forecast of dengue incidence using temperature and rainfall. PLoS Negl Trop Dis 6(11):e1908

Hoeck PA, Ramberg FB, Merrill SA, Moll C, Hagedorn HH (2003) Population and parity levels of Aedes aegypti collected in Tucson. J Vector Ecol 28(Tempelis 1975):65-73

Hurvich CM, Tsai CL (1991) Bias of the corrected AIC criterion for under-fitted regression and time series models. Biometrika 78(3): 499-509. https://doi.org/10.1093/biomet/78.3.499

Khalid B, Ghaffar A (2015a) Dengue transmission based on urban environmental gradients in different cities of Pakistan. Int J Biometeorol 59(3):267-283

Khalid B, Ghaffar A (2015b) Environmental risk factors and hotspot analysis of dengue distribution in Pakistan. Int J Biometeorol 59(11):1721-1746

Kutsuna S, Kato Y, Moi ML, Kotaki A, Ota M, Shinohara K, Kobayashi T, Yamamoto K, Fujiya Y, Mawatari M, Sato T, Kunimatsu J, 
Takeshita N, Hayakawa K, Kanagawa S, Takasaki T, Ohmagari N (2015) Autochthonous dengue fever, Tokyo, Japan, 2014. Emerg Infect Dis 21(3):517-520

Lai S, Huang Z, Zhou H, Anders KL, Perkins TA, Yin W, Li Y, Mu D, Chen Q, Zhang Z, Qiu Y, Wang L, Zhang H, Zeng L, Ren X, Geng M, Li Z, Tatem AJ, Hay SI, Yu H (2015) The changing epidemiology of dengue in China, 1990-2014: a descriptive analysis of 25 years of nationwide surveillance data. BMC Med 13:100

Morales I, Salje H, Saha S, Gurley ES (2016) Seasonal distribution and climatic correlates of dengue disease in Dhaka, Bangladesh. Am J Trop Med Hyg 94(6):1359-1361

Morin CW, Comrie AC, Ernst K (2013) Climate and dengue transmission: evidence and implications. Environ Health Perspect 121(1112):1264-1272

Mutheneni SR, Morse AP, Caminade C, Upadhyayula SM (2017) Dengue burden in India: recent trends and importance of climatic parameters. Emerg Microbes Infect 6(8):e70

Nagao Y, Koelle K (2008) Decreases in dengue transmission may act to increase the incidence of dengue hemorrhagic fever. Proc Natl Acad Sci 105(6):2238-2243

Seidahmed OME, Eltahir EAB (2016) A sequence of flushing and drying of breeding habitats of Aedes aegypti (L.) prior to the low dengue season in Singapore. PLoS Negl Trop Dis 10(7):e0004842

Shepard DS, Undurraga EA, Halasa YA (2013) Economic and disease burden of dengue in Southeast Asia. PLoS Negl Trop Dis 7(2): e2055

Shi Y, Liu X, Kok SY, Rajarethinam J, Liang S, Yap G, Chong CS, Lee KS, Tan SS, Chin CK, Lo A, Kong W, Ng LC, Cook AR (2016) Three-month real-time dengue forecast models: an early warning system for outbreak alerts and policy decision support in Singapore. Environ Health Perspect 124(9):1369-1375. https://doi. org/10.1289/ehp.1509981
Stone M (1974) Cross-validatory choice and assessment of statistical predictions. J R Stat Soc Ser B 36(2):111-147

Trippa L, Waldron L, Huttenhower C, Parmigiani G (2015) Bayesian nonparametric cross-study validation of prediction methods. Ann Appl Stat 9(1):402-428

Wang SF, Chang K, Loh EW, Wang WH, Tseng SP, Lu PL, Chen YH, Chen YMA (2016) Consecutive large dengue outbreaks in Taiwan in 2014-2015. Emerg Microbes Infect 5(12):e123

Wearing HJ, Rohani P (2006) Ecological and immunological determinants of dengue epidemics. Proc Natl Acad Sci 103(31):1180211807

World Health Organization (2009) Dengue guidelines for diagnosis, treatment, prevention and control: new edition

World Health Organization (2017) Dengue and dengue haemorrhagic fever WHO fact sheet $\mathrm{N}^{\circ} 117$

Xu L, Stige LC, Chan KS, Zhou J, Yang J, Sang S, Wang M, Yang Z, Yan Z, Jiang T, Lu L, Yue Y, Liu X, Lin H, Xu J, Liu Q, Stenseth NS (2017) Climate variation drives dengue dynamics. Proc Natl Acad Sci 114(1):113-118

Yang HM, Macoris MLG, Galvani KC, Andrighetti MTM, Wanderley DMV (2009a) Assessing the effects of temperature on dengue transmission. Epidemiol Infect 137(08):1179-1187

Yang HM, Macoris MLG, Galvani KC, Andrighetti MTM, Wanderley DMV (2009b) Assessing the effects of temperature on the population of Aedes aegypti, the vector of dengue. Epidemiol Infect 137(08):1188-1202

Yu HL, Lee CH, Chien LC (2016) A spatiotemporal dengue fever early warning model accounting for nonlinear associations with hydrological factors: a Bayesian maximum entropy approach. Stoch Env Res Risk A 30(8):2127-2141 